


\title{
Caracterização gravimétrica da Zona de Fratura São Paulo
}

Roberta Ladislau de Medeiros, IAG-USP (peridotito@iag.usp.br) e Eder C. Molina (eder@iag.usp.br)

Copyright 2008, SBGf - Sociedade Brasileira de Geofísica

Este texto foi preparado para a apresentação no IV Simpósio Brasileiro de Geofísica, Belém, 14 a 17 de novembro de 2010. Seu conteúdo foi revisado pelo Comitê Técnico do IV SimBGf, mas não necessariamente representa a opinião da SBGf ou de seus associados. É proibida a reprodução total ou parcial deste material para propósitos comerciais sem prévia autorização da SBGf.

\section{Resumo}

A investigação da Terra através da gravimetria implica um grande número de informações sobre o seu interior em diferentes escalas de observação. O presente estudo considerou pesquisas anteriormente realizadas na área da Zona de Fratura São Paulo e Arquipélago São Pedro e São Paulo, no Oceano Atlântico Equatorial, cujos dados geoquímicos e petrológicos apontam a presença de uma anomalia térmica. A fim de caracterizar através da gravimetria a Zona de Fratura São Paulo utilizaram-se dados de gravidade, anomalia ar livre altura geoidal e tensor gradiente de gravidade.

\section{Introdução}

As falhas transformantes são caracterizadas pelo movimento extensional. Estas falhas seccionam a dorsal mesoceânica em eixos onde estão localizados os centros de espalhamento do assoalho oceânico. As zonas de fratura englobam a porção sismicamente ativa e seu traço fóssil formado por um desnível batimétrico e inativo (Fowler, C.M.R. 2005).

O termo mullion está descrito na literatura denominando feições topográficas localizadas na região de entrucamento ridge-falha transformante. Já o termo megamullion é aplicado às feições cujo diâmetro é maior do que $10 \mathrm{~km}$ (Tucholke et al. 2001). O Arquipélado São Pedro São Paulo (ASPSP) é descrito como um megamullion localizado na Zona de Fratura São Paulo (ZFSP) (Sichel et al, 2008).

Nesta região, existe um debate acerca da origem de cold spot (anomalia térmica negativa) presente no manto. Especula-se que a temperatura do manto abaixo do Atlântico equatorial seria menor cerca de $150^{\circ} \mathrm{C}$ do que em qualquer outra área da dorsal meso-atlântica (Bonatti et al., 1992, 1993 apud Schilling et al., 1995).

Bonatti (1990) foi pioneiro na investigação do cold spot. Ele comparou às rochas encontradas nas Ilhas Zabargad (Mar Vermelho) as encontradas no ASPSP cujos peridotitos milonitizados (grãos que apresentam dimensões variadas) sofreram processo semelhante de serpentinização (metamorfismo de baixo grau com hidratação dos minerais). Bonatti sugeriu a ocorrência de metassomatismo (injeção de fluídos nas atuais zonas de cisalhamento) reduzindo a temperatura. Entretanto, Schilling e colaboradores (1987) a bordo do navio de pesquisa Robert Conrad mapearam a distribuição de idade da crosta oceânica, realizaram análises geoquímicas, acompanharam a atividade magmática do local através de sonar de varredura lateral (GLORIA) levantaram a hipótese de aprisionamento de slab devido aos processos de agregação e dispersão de continentes no decorrer do tempo geológico. Esta hipótese é baseada na reconstrução paleogeográfica elaborada por Daziel (1997) a qual descreve eventos de subducção anteriores a formação de Pangéia ocorridos na região equatorial do que hoje é o atual Oceano Atlântico (Sichel et al. 2008).

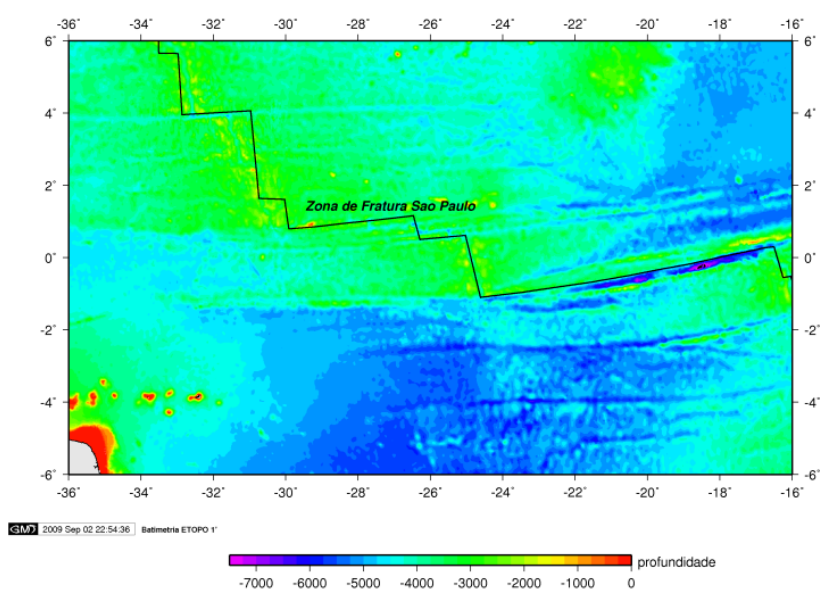

Figura 1 - Localização da área de estudo.

\section{Metodologia/ Problema Investigado}

A forma do geóide está diretamente relacionada ao campo de gravidade da Terra. Trata-se de uma superfície equipotencial que corresponde ao nível médio dos mares, excetuando perturbações, prolongado sobre os continentes. A forma do elipsóide está relacionada à superfície matemática cujos parâmetros variam conforme a área considerada. A diferença entre o geóide e 0 elipsóide de revolução denomina-se altura ou ondulação geoidal sendo representado por $\mathrm{N}$. Alturas geoidais encontram-se relacionadas a regiões de subducção, a presença de slab e presença de hotspots. Anomalias geoidais de longo comprimento indicam uma contribuição elevada dos graus menores do modelo gepotencial. Referente à fontes profundas no manto. Enquanto, as anomalias de curto comprimento indicam uma maior contribuição dos graus mais elevados do modelo geopotencial. Referente à fontes mais rasas localizadas na crosta e no manto superior (Bowin, 1985). Os dados 
de altura geoidal residual foram obtidos através do Modelo Geopotencial 2008 (Earth Geopotencial Model 2008) (Pavlis et al., 2008). Estes valores foram calculados a partir de um conjunto de programas disponibilizados pela Universidade de Trieste (Itália) em parceria com a Universidade de Tecnologia da Eslováquia. Os programas grafim.job, e g2grd_grafim.exe realizaram o cálculo da anomalia ar livre e altura geoidal (M. Šprlák and J. Janák, 2006). As anomalias de geóide residual foram calculadas a partir da subtração das grades obtidas pelo modelo EGM08. Obtendo-se os seguintes graus: 10 a 2160,15 a 2160, 20 a 2160, 25 a 2160, 30 a 2160 e 35 a 2160. Da mesma forma obtiveram-se valores de anomalia de ar livre residual para estes mesmos graus. Os dados de batimetria foram obtidos a partir do banco de dados geofísicos Geodas. Disponibilizado no banco de dados geofísicos da NOAA Considerando o estudo pioneiro de Haxby e Turcotte (1978) cujo modelo térmico isóstatico aplicado a dorsal mesoatlântico indicou que os dados de altura geoidal recebem contribuições de muitas fontes diferentes tais como parâmetros termais de resfriamento de placas litosféricas, batimetria, idade da litosfera etc (Chaise et al., 1985) Foi realizada a análise da correlação (Coeficientes de Person) entre os dados de batimetria e de altura geoidal residual. Encontrou-se uma correlação positiva que indicou a influência das massas topográficas sobre os valores de altura geoidal encontrados. Desta forma, a fim de estimar este efeito, utilizou-se um programa que simulou a presença da topografia sobre o geóide através da construção de prismas (cujas densidades variaram entre 2900 a $1030 \mathrm{~kg} / \mathrm{m}^{3}$ ). O programa prisma3D (Chaves, 2007) forneceu uma grade de altura geoidal influenciada pela topografia. De acordo com a teoria flexural, a placa litosférica apresenta um comportamento elástico em sua porção mais superficial. Se uma carga é "colocada" sobre esta placa ela irá flexionar-se. Geralmente, placas mais jovens tendem a apresentar valor de Te mais baixo. Placas mais antigas, como litosferas cratônicas, tendem a apresentar um valor de Te mais alto. De acordo com Parker (1973) existe uma relação entre a flexura da placa e anomalia de gravidade. Utilizou-se o programa Admgravitylayred (Chaves, 2008) a fim de estimar este efeito. Foram calculadas as grades de gravidade subtraindo-se este efeito. A partir destes cálculos foram plotados perfis indicando à contribuição da topografia a anomalia observada. Para utilizar uma ferramenta que pudesse confirmar esta proposição utilizou-se o programa TGG (Santos, 2007) para calcular o tensor gradiente da gravidade. Indicando a taxa de variação dos componentes do vetor segundo as direções $\mathrm{XYZ \text {. }}$

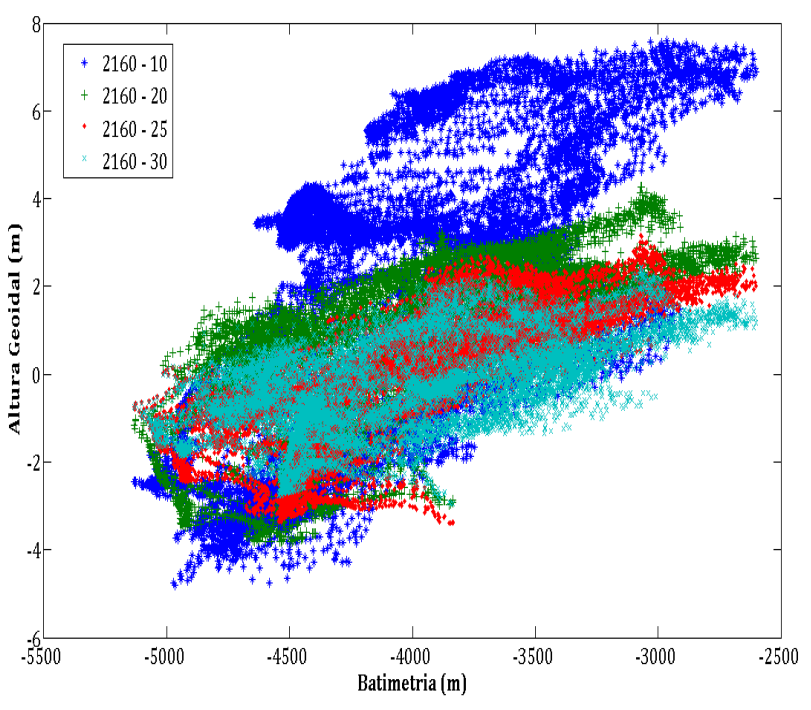

Figura 2 - Análise de correlação. Eixo y: altura geoidal residual. Eixo $x$ : batimetria

\section{Resultados}

Observando os perfis a seguir de altura geoidal e altura geoidal residual é possível visualizar a proximidade entre a ondulação positiva e as características batimétricas da cordilheira mesoceânica. A anomalia geoidal destacou-se a partir do grau 20 com cerca de 3 metros. Tornando-se mais intensa até desaparecer no grau 40. Esta proposição é ressaltada a partir de uma análise dos dados do tensor gradiente de gravidade. Pode-se observar a variação da gravidade na direção OX (gxz) e variação vertical da gravidade (gzz) onde se destacam as feições batimétricas. Pode-se concluir que qualquer investigação com base em gravimetria na área da ZFSP precisa considerar os efeitos do intenso fraturamento na crosta e os processos magmáticos presentes assim como os efeitos da compensação isostática na placa oceânica. Devido à proximidade a dorsal, as análises de altura geoidal, gravidade e anomalia ar livre indicaram a presença marcante no campo geopotencial dos processos tectono-magmáticos existentes em centros de espalhamento.

\section{Discussão e Conclusões}

Diversos trabalhos realizados anteriormente na região basearam suas análises em dados petrológicos e geoquímicos. Entretanto, para obter maiores informações a respeito desta anomalia térmica é pertinente a utilização de outros métodos geofísicos. Modelos tomográficos globais e análise de fluxo térmico poderão contribuir para a análise da área a fim de delimitar as características do manto superior na região. 


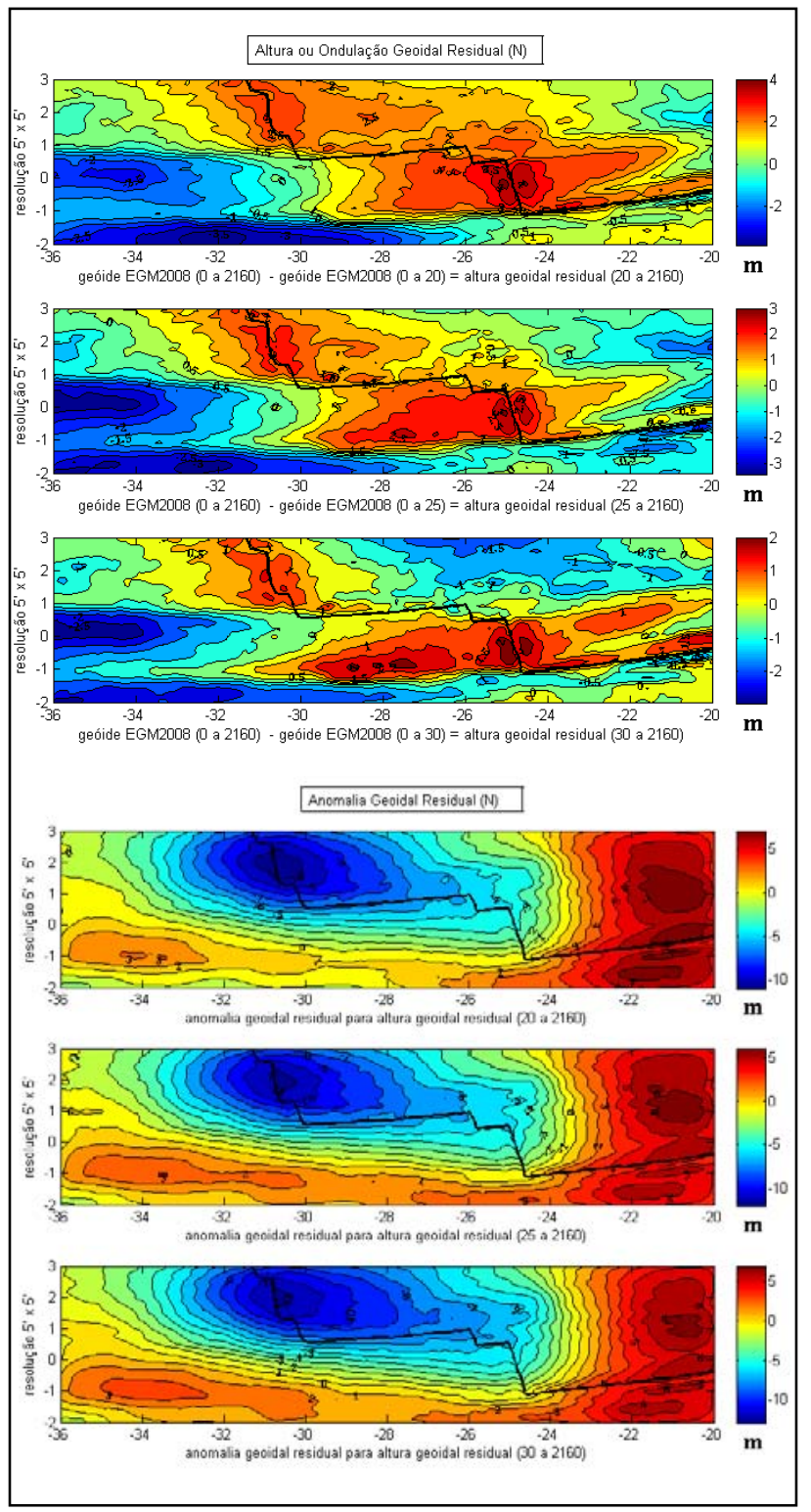

Figura 3 - Mapas de altura geoidal residual e Anomalia Geoidal Residual.

\section{Agradecimentos}

Agradeço a Coordenação de Aperfeiçoamento do Ensino Superior pela bolsa concedida. Agradeço aos amigos Carlos Moreno e Henrique Bueno pela disponibilização dos programas.

\section{Referências}

Agapova, C.V. Morfology of the eastern active segment of the St. Paul fracture zone (The Equatorial Atlantic). Oceanology, America Geophysical Union. English translation, vol. 34, n. 1, pp. 99-103, 1994.

Anderson, D.L. Phase changes and Mantle Mineralogy In:_. Theory of the earth. Blackwell Scientific Publication. pp. 337-353, 1989.

Amos, M.J., Featherstone, W.E. Comparisons of recent global gepotential models with terrestrial gravity field data over New Zealand and Australia. Geomatics Research Australasia. 2003. Disponível em:<http://www.cage.curtin.edu.au/ will/Featherstone_G RA_78_67-84.pdf>. Acesso em: 15 jun. 2009.

Asmus, H.E., Guazelli, W. Descrição sumária das estruturas da margem continental brasileira e das áreas oceânicas e continentais, adjacentes. Hipótese do tectonismo causador, e implicações para os prognósticos do potencial de recursos minerais. Série Projeto REMAC, n. 9, Petrobras, Rio de Janeiro, pp.187-261, 1981.

Beltrão, J.F., Silva, J.B.C., Costa, J.C. Robust Polynomial Fitting Method for Regional Gravity Estimation. Geophysics. 1991. Disponível em: $<$ http://scitation.aip.org/dbt/dbt.jsp?KEY=GPYSA7\&Volum e=56\&lssue=1>. Acesso em: 10 jul. 2009.

Blakely, R. J. Potential Theory in Gravity and Magnetic Applications. Cambridge University Press. pp. 411. 1996.

Bonatti, E. O Manto sob os oceanos. As formas mutantes da Terra. Scientific American Brasil. Edição Especial. n. 20. Outubro. 2007.

Bonatti, E. Anomalous opening of the Equatorial Atlantic due to an equatorial mantle thermal minimum. Earth and Planetary Science Letters. vol. 250, n. 4977, pp. 107111, 1996.

Bonatti, E, Seyler, M. and Sushevskaya, N. A cold suboceanic mantle belt at the Earth's equator. Science, vol. 261, n. 5119, pp. $315-320,1993$.

Bonatti, E, Peyve, A., Kepezhinskas, P., Kurentsova, N., Seyler, M. Skolotnev, I. S., and Udintsev, G. Upper Mantle Heterogeneity Below the Mid-Atlantic Ridge $0^{\circ}-$ $15^{\circ}$ N. J. Geophys. Res., vol. 97, n. B4, pp. 4461-4476, 1992. 
Bonatti, E. Subcontinental mantle exposed in the Atlantic Ocean on St. Peter-Paul islets. Nature. 1990.

Disponível em:

<http://www.nature.com/nature/journal/v345/n6278/pdf/34 5800a0.pdf>. Acesso em: 15 jul. 2008.

Bonatti, E. Not so hot "hot spots" in the oceanic mantle. Science. vol. 250. n. 4977, pp. 107 -111, 1990.

Bowin, C. Topography at the core-mantle boundary. Geophysical Research Letters, vol. 13, n. 13, pp. 15131516, 1986.

Campos T.F.C., Virgens Neto, J. das, Sirivastava, N.K., Petta, R.A., Hartmann, L.A., Moraes J.F.S. de, Mendes L., Silveira S.R.M. Arquipélago de São Pedro e São Paulo. Soerguimento tectônico de rochas infracrustais no Oceano Atlântico. 2005. Disponível em: $<$ http//www.unb.br/ig/sigep/sitio 002/sitio 002.pdf>. Acesso em: 15 jul. 2008.

Chaves, C. A. M., Detectabilidade de plumas mantélicas profundas a partir de anomalias do geóide. Trabalho de graduação do curso de Bacharelado em Geofísica. Universidade de São Paulo, São Paulo, pp. 36, 2007.

Daziel, I.W.D. A Terra muito antes da Pangea. As formas mutantes da Terra. Scientific American Brasil. Edição Especial. n. 20. Outubro. 2007.

Daziel, I.W.D. Neoproterozoic-Paleozoic geography and tectonics: Review, hypothesis, environmental speculation. GSA Bulletin. vol. 109, n.1, pp. 16-42, 1997.

Equatorial Segment of the Mid-Atlantic Ridge (EQUARIDGE). 1996. Initial Results of the Geological and Geophysical Investigations under the EQUARIDGE Program. Cruises of $r / v$ 'Akademik Nikolaj Strakhov' in 1987, 1990, 1991. Intergovernmental Oceanographic Commission Technical Series. Unesco. pp. 128.

Fowler, C.M.R. Past Plate Motions. In: The Solid Earth. An Introduction to Global Geophysics. Cambridge University Press. pp. 43-99, 2005.

Hager, B.H. Subducted slabs and the geoid constraints on mantle rheology and flow. Journal of Geophysical Research, vol. 89, n. B7, pp. 6003-6015, 1984.

Hekinian, R., Juteau, T., Gracia, E., Sichler, B., Sichel, S., Udintsev, G., Apprioual, R. and Ligi, M. Submersible observations of Equatorial Atlantic mantle: The St. Paul Fracture Zone region. Marine Geophysical Researches. vol. 21, pp. 529-560, 2000.

National Geospacial Intelligency Agency. Description of Files Related to using the EGM2008 Global Gravitational Model to compute Geoid Undulations with respect to WGS84. Disponível em: <http://earthinfo.nga.mil/GandG/wgs84/gravitymod/egm2008/READM E_WGS84_2.pdf>. Acesso em: 10 set. 2009.
Mello, S. L. M. Tectonics and Volcanism of the MidAtlantic Ridge at $45^{\circ} \mathrm{N}$. Tese de Doutoramento. University of Leeds, Leeds, pp. 200, 1999.

NGDC (NATIONAL GEOPHYSICAL DATA CENTER). Disponível em:

<http://map.ngdc.noaa.gov/website/mgg/trackline/viewer. htm>. Acesso em: 15 jul. 2008.

Molina, E. C. Ajustamento e integração de dados gravimétricos e de altimetria por satélite na representação do campo de gravidade no Atlântico Sul. Tese de doutoramento. Universidade de São Paulo, São Paulo, pp. 201, 1996.

Moraes, J. F. S. de. Caracterização Petrográfica e Química das Rochas do Arquipélago de São Pedro e São Paulo. Companhia de Pesquisas de Recursos Minerais. Superintendência Regional de Recife. 1996.

Palma, J. J. C. Morfotectônica e gravimetria da falha transformante de Ascensão e crista da Cordilheira Mesoatlântica de $6^{\circ} 20^{\prime}$ S a $10^{\circ} 05^{\prime}$ S. São Paulo. Tese de doutorado. Universidade de São Paulo, São Paulo, pp. 106, 1998.

Pavlis, K. N., Holmes, S. A., Kenyon, S. C. and Factor, J. K. 2008. An Earth Gravitational Model to Degree 2160: EGM2008. 2008. Austria. Disponível em:<http://www.dgfi.badw.de/typo3_mt/fileadmin/2kolloqu ium_muc/2008-10-08/Bosch/EGM2008.pdf>. Acesso em: 15 jü. 2008.

Parker, R.L. The Rapid Calculation of potencial Anomalies. Geophys. J.R. astr. Soc. 31,p. 447-455, 1973.

Press, F., Siever, R., Grotzinger, J., Jordan, T.H. A Evolução dos Continentes. In: Para Entender a Terra. $4^{\mathrm{a}}$ edição. Bookman. pp. 195-226, 2006.

Relatório técnico produzido pela Universidade de Bretagne Ocidental, IFREMER e LAGEMAR, 1996.

Saatkamp, E. D., Moraes, C. V. de, Farret, J.C. Avaliação do modelo geoidal EGM2008 para o Brasil.2009. Anais do XIV Simpósio Brasileiro de Sensoriamento Remoto, Natal, Brasil, 2009, pp. 4303-4310.

Šprlák, M., Janák, J.: Gravity field modeling. New program for gravity field modeling by spherical harmonic functions. p.1-8,2006.

Schilling, J.G., Ruppel, C. Davis, A. N., McCully, B., Tighe, S.A., Kingsley, R. H. and Lin, J. Thermal structure of the mantle beneath the equatorial Mid-Atlantic Ridge: Inferences from the spatial variation of dredged basalt glass composition. Journal of Geophysical Research. vol. 100, n. B7. pp. 10.057-10.076, 1995.

Sichel, S. E., Motoki. A., Esperança, S, Horan, M. F., Maia, M., Szatmari, P., Mello, S L.M. and Alves, E. C. 2008. Geophysical and geochemical evidence for cold 
upper mantle beneath the Equatorial Atlantic Ocean. Revista Brasileira de Geofísica. vol. 26, n. 1, pp. 69-86, 2008.

Sperle, M., Mello, S.L.M., Costa, M.P.A. Modelagem gravimétrica e magnética do limite de crostas continental/oceânica no plateau do Rio Grande do Norte. 1989. Anais do I Congresso Internacional da SBGF. Rio de Janeiro, vol. 3, pp. 67-72.

Stanton, N., Mello, S. L., Sichel, S. E. 2006. Morfoestrutura da Cordilheira Mesoceânica no Atlântico Sul entre $0^{\circ} \mathrm{S}$ e $50^{\circ} \mathrm{S}$. Revista Brasileira de Geofísica Vol. 24(2). 231-241 pp.

Talwani, M., Worzel, J.L. and Landisman, M. Rapid Gravity Computations for Two-dimensional Bodies with Applications to the Mendocino Submarine Fracture Zone. Journal of Geophysical Research, vol. 64, pp. 49-59, 1959.

Tucholke, B.E., Fujoka, K., Ishihara, T., Hirth, G. and Kinoshita, M. Submersible study of na oceanic megamullion in the central North Atlantic. Journal of Geophysical Research, vol. 106, n. B8, pp. 145-161, 2001.

Tucholke, B.E., Lin, J. and Kleinrock, M. C. Megamullion and mullion structure defining oceanic metamorphic core complexes on the Mid-Atlantic Ridge. Journal of Geophysical Research, vol.103, n. B5, pp. 9857-9866, 1998.

Wessel, P. and Watts, A. B. On the accuracy of marine gravity measurements. Journal of Geophysical Research, vol. 93, pp. 393- 413. 1988. 



Figura 4 - Mapas dos componentes do tensor gradiente da gravidade. Calculados a partir do programa TGG.m Resolução 5' x 5' 